\title{
Deep learning for enhancing multi-source reverse time migration
}

This paper was downloaded from TechRxiv (https://www.techrxiv.org).

LICENSE

CC BY-NC-SA 4.0

SUBMISSION DATE / POSTED DATE

30-12-2021 / 05-01-2022

CITATION

Li, Yaxing; Jia, Xiaofeng; Wu, Xinming; Geng, Zhicheng (2022): Deep learning for enhancing multi-source reverse time migration. TechRxiv. Preprint. https://doi.org/10.36227/techrxiv.17704505.v1

$\mathrm{DOI}$

10.36227/techrxiv.17704505.v1 


\title{
Deep learning for enhancing multi-source reverse time migration
}

\author{
Yaxing Li, Xiaofeng Jia, Xinming Wu and Zhicheng Geng
}

\begin{abstract}
Reverse time migration (RTM) is a technique used to obtain high-resolution images of underground reflectors; however, this method is computationally intensive when dealing with large amounts of seismic data. Multi-source RTM can significantly reduce the computational cost by processing multiple shots simultaneously. However, multi-source-based methods frequently result in crosstalk artifacts in the migrated images, causing serious interference in the imaging signals. Plane-wave migration, as a mainstream multi-source method, can yield migrated images with plane waves in different angles by implementing phase encoding of the source and receiver wavefields; however, this method frequently requires a trade-off between computational efficiency and imaging quality. We propose a method based on deep learning for removing crosstalk artifacts and enhancing the image quality of plane-wave migration images. We designed a convolutional neural network that accepts an input of seven plane-wave images at different angles and outputs a clear and enhanced image. We built $5051024 \times 256$ velocity models, and employed each of them using plane-wave migration to produce raw images at $0^{\circ}, \pm 20^{\circ}, \pm 40^{\circ}$, and $\pm 60^{\circ}$ as input of the network. Labels are high-resolution images computed from the corresponding reflectivity models by convolving with a Ricker wavelet. Random sub-images with a size of $512 \times 128$ were used for training the network. Numerical examples demonstrated the effectiveness of the trained network in crosstalk removal and imaging enhancement. The proposed method is superior to both the conventional RTM and plane-wave RTM (PWRTM) in imaging resolution. Moreover, the proposed method requires only seven migrations, significantly improving the computational efficiency. In the numerical examples, the processing time required by our method was approximately $1.6 \%$ and $10 \%$ of that required by RTM and PWRTM, respectively.
\end{abstract}

\section{INTRODUCTION}

Seismic exploration is a geophysical method to infer the geometries and properties of underground rocks by analyzing the propagation pattern of seismic waves generated by artificial seismic sources at the surface. This method is regarded as one of the most effective methods to explore oil and gas resources. Complex underground structural imaging has become particularly valuable in recent years with increasing resource extraction; therefore, acquiring high quality seismic data and developing accurate imaging algorithms has gained importance. Although wide- and full-azimuth acquisition techniques can yield adequate subsurface reflection signals, these massive data also entail a huge computational overhead [1] [2]. Seismic migration is an imaging technique that returns the

Y. Li, X. Jia, and X. Wu are with the Laboratory of Seismology and Physics of Earth's Interior, School of Earth and Space Sciences, University of Science and Technology of China, Hefei, China and National Geophysical Observatory at Mengcheng, University of Science and Technology of China, Anhui, China. Zhicheng Geng is with the Bureau of Economic Geology, University of Texas at Austin, Austin, TX 78713, USA. (Email: xjia@ustc.edu.cn). reflections or diffractions from data to their true subsurface locations, constructing a high-resolution image of underground structures [3]. Several migration methods have been developed and applied in earlier studies [4] [5], for example, Kirchhoff migration [6] [7] [8] and Gaussian-beam migration [9] [10] based on ray theory, generalized screen propagators [11] [12] based on one-way wave equation, and reverse time migration (RTM) [13] based on two-way wave equation.

RTM, one of the most accurate migration methods, utilizes the full seismic wavefields and is capable of handling arbitrarily complex media with amplitude fidelity [5] [14]. Figure 1 illustrates the workflow for executing RTM. We start by extracting the seismic records as common-shot gathers and preparing a migration velocity model. Subsequently, we execute the shot loop. For each shot, we need to forwardextrapolate the source wavefield and backward-extrapolate the receiver wavefield by loading the source and corresponding data, respectively. The zero-time lag cross-correlation imaging condition applied on the two wavefields generates one singleshot image. After the shot loop is finished, all the single-shot images are stacked to obtain the final imaging result. However, in this process, the propagation of the wavefields is extremely time-consuming. Assuming $N$ sources, a total of $N$ times of migration is required, corresponding to $2 N$ times of wavefields propagation, which includes $N$ times each of both forward and backward wavefields propagation. The computational burden increases significantly with increasing number of shots.

To reduce the computational cost of RTM, several multisource RTM methods have been proposed and developed [15] [16] [17] [18] [19] [20]. Unlike conventional RTM that deals with each shot separately, multi-source migration methods focus on forming a virtual source and the corresponding virtual shot gather by weighted summing multiple shots and their corresponding shot gathers simultaneously. The virtual shot and its corresponding shot gather are migrated to generate an image. Multi-source RTM reduces the number of migrations significantly, thus improving computational efficiency. However, a serious drawback of these multi-source methods is that mismatched sources and shot gathers produce crosstalk artifacts under nonlinear imaging conditions [18], severely hurting the imaging quality. Various researches have been conducted to suppress the crosstalk by designing different coding functions. Such encoding methods are primarily classified into phase and amplitude encoding. Numerous scientists have investigated phase coding methods [21] [22] [19] [23], wherein they concentrated on shifting the crosstalk positions in the migrated images to suppress them. To date, several types of phase coding methods have been developed, such as plane- 


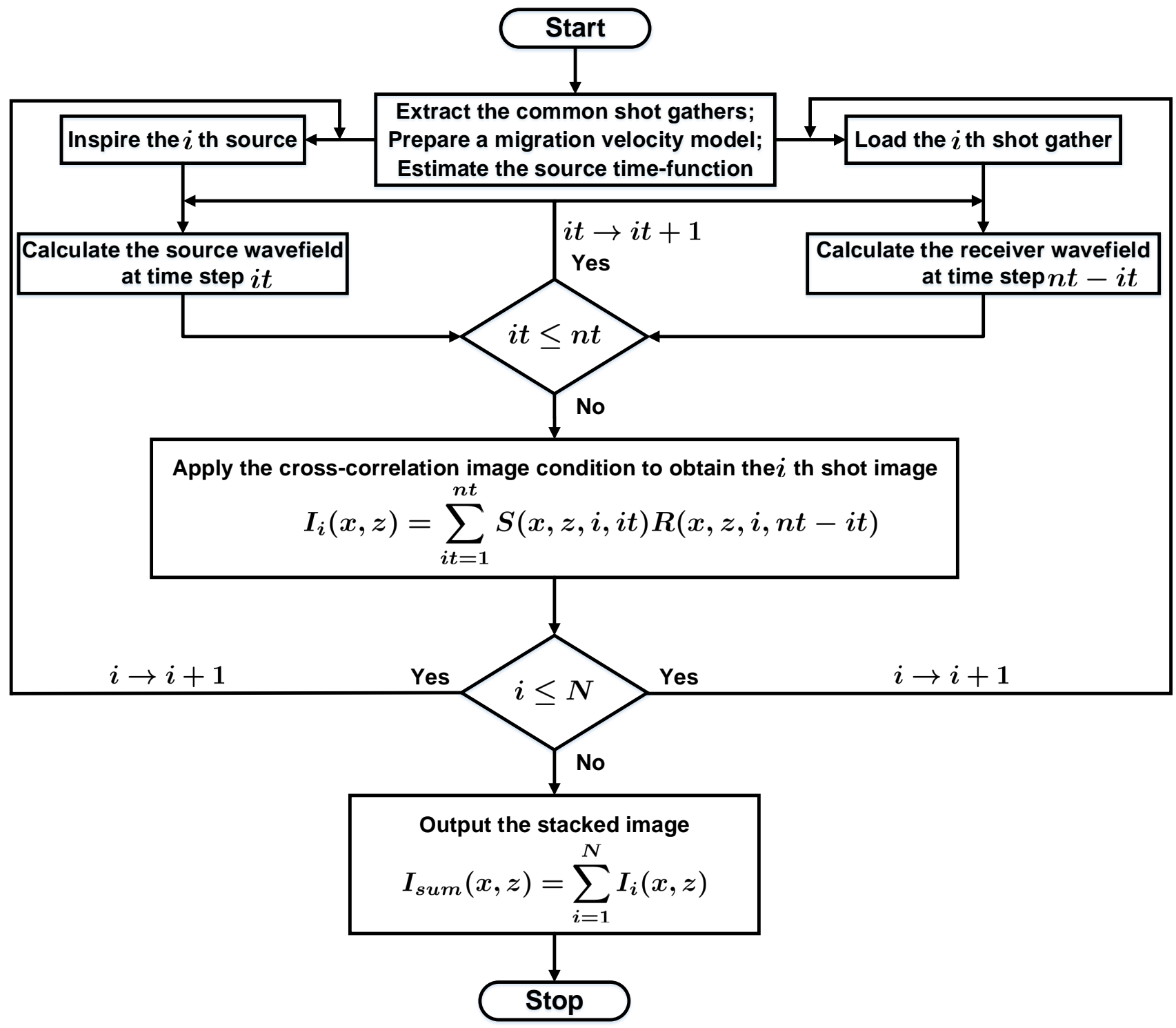

Figure 1: RTM execution workflow. Here $i$ denotes the shot number, it denotes the time step, and $n t$ is the total time steps. $S(x, z, i, i t)$ and $R(x, z, i, i t)$ represent the source and receiver wavefields of the $i$ th source at time step it, respectively.

wave encoding, random phase encoding, modulation encoding, and harmonic encoding. Among them, the plane-wave RTM (PWRTM) is the most widely deployed multi-source migration method. In PWRTM, different linear time delays imposed on the source and the receiver wavefields yield planar wavefields with different angles. The cross-correlation imaging condition is placed on all the angular planar wavefields to generate the corresponding single-angle images, which are called commonangle image gathers (CAIGs). PWRTM images are created by stacking the CAIGs. The time delay and the number of angles affect the computational efficiency. A few research works based on amplitude encoding [16] [17]. In these methods, the imaging results of several different encoding functions are stacked to accomplish signal enhancement and noise suppression [16]. Jia et al. (2020) presented a method for simultaneously encoding the phase and amplitude [24]. This technique involves fitting the wavefield of a virtual supershot in the time domain by encoding the amplitude and phase of the wavefields excited by multiple sources at the surface. A dictionary learning approach was proposed to mute the crosstalk noise in least-squares RTM [25]. Although multisource RTM-based methods have made great progress, they involve a trade-off between computational efficiency and suppression of noise. There is an urgent demand for a method that can guarantee a significant increase in computational efficiency while eliminating crosstalk noise.

Deep learning has advanced rapidly in the field of computer vision in recent years owing to the widespread use of convolutional neural networks (CNNs). CNN-based methods have progressed substantially in image classification [26], segmentation [27], object detection [28] and denoising [29] tasks. Compared with traditional methods, deep learning does not require manually defined criteria and automatically picks up the deep-level information of images. Deep learning has been successfully employed for data processing and interpretation in seismic exploration, for example, seismic 
data noise attenuation [30] [31] [32] [33], first-arrival picking [34] [35] [36], velocity model building [37] [38] [39], impedance inversion [40] [41], and seismic structural interpretation [42] [43] [44]. Although, a few advances in deep learning have been reported for simultaneous-source data deblending [45] [46] [47], few studies have used deep learning for image denoising in multi-source seismic migration.

We propose a CNN to eliminate the crosstalk in the migrated images and enhance the PWRTM image quality. The input of the network are common-angle image gathers (CAIGs), that is, a combination of plane-wave images with seven incident angles $\left(0^{\circ}, \pm 20^{\circ}, \pm 40^{\circ}\right.$, and $\left.\pm 60^{\circ}\right)$. The input has seven channels. The desired output is a clear and high-resolution image convolved from the corresponding reflectivity model, and we called it 'convolved image'. The designed network architecture is a modified U-net network [48] with three parts: an encoder, decoder, and refinement module. The encoder learns the features of input noisy image gathers at different levels. The decoder restores the high-level feature map to the same size as that of the input images. The refinement module predicts a clear image. To generate rich training data, we began by building 505 two-dimensional (2D) velocity models with different types of structures. With these velocity models, we placed the source and geophones at the surface to simulate the corresponding synthetic seismic records using the finite difference method. A slight smoothing was conducted on these velocity models, and the smoothed velocity models were employed for PWRTM to obtain CAIGs. Labels (highresolution convolved images) were built by true velocity models. We defined a Laplacian pyramid- $\mathcal{L} 1$ loss function for training the network. Using the training data and network architecture, we trained the CNN model with the defined loss function. Through numerical examples, we compared the stacked images of input CAIGs (i.e., the PWRTM images), predicted results, and ground truths. The comparisons revealed that the trained model realizes crosstalk removal and improves the imaging resolution. In addition, we tested the model with complex structures and compared the results for a stacked image of input CAIGs (i.e., simplified PWRTM image with only seven angles), traditional RTM image with 277 shots, and PWRTM image with 77 angles in terms of the image quality and processing time.

\section{THEORY}

The 2D first-order velocity-stress equation in the acoustic medium is written as

$$
\left\{\begin{array}{l}
\frac{\partial v_{x}}{\partial t}=-\frac{1}{\rho} \frac{\partial p}{\partial x} \\
\frac{\partial v_{z}}{\partial t}=-\frac{1}{\rho} \frac{\partial p}{\partial z} \\
\frac{\partial p}{\partial t}=-\rho c^{2}\left(\frac{\partial v_{x}}{\partial x}+\frac{\partial v_{z}}{\partial z}\right) .
\end{array}\right.
$$

Here $v_{x}$ and $v_{z}$ denote the $\mathrm{x}$ - and z-components of particle velocity, respectively; $p$ is the pressure wavefield. $\rho$ and $c$ indicate the density and acoustic velocity of the media, respectively. In the conventional RTM, the source wavefield $p^{S}(x, z, i, t)$ of shot $i$ is cross-correlated with its corresponding receiver wavefield $p^{R}(x, z, i, t)$, and we can obtain a singleshot image as follows:

$$
I(x, z, i)=\sum_{t=0}^{T} p^{S}(x, z, i, t) p^{R}(x, z, i, t),
$$

where $T$ represents the recording time. Summing all the single-shot images, we obtain a stacked image $I_{\text {sum }}(x, z)$, represented by

$$
I_{\text {sum }}(x, z)=\sum_{i=1}^{N} \sum_{t=0}^{T} p^{S}(x, z, i, t) p^{R}(x, z, i, t),
$$

where $N$ is the number of shots. For this method, we need $N$ forward extrapolations of the source wavefield and $N$ backward extrapolations of the receiver wavefield, leading to a total of $2 N$ simulation cycles. As the number of shots increases, the computational consumption of the conventional RTM is enormous.

For the plane-wave RTM, the source and receiver wavefields of each shot were first encoded by time delay, and the encoded source and encoded receiver wavefields of all shots were respectively stacked to obtain the corresponding stacked wavefields. Various time delay patterns form different incidentangle wavefields. The stacked source $p_{\text {sum }}^{S}$ and stacked receiver wavefields $p_{\text {sum }}^{R}$ at the incident angle of $\theta$ respectively assume the form

$$
\left\{\begin{array}{l}
p_{\text {sum }}^{S}(x, z, t, \theta)=\sum_{i=1}^{N} p^{S}(x, z, i, t) * \delta\left(t-f\left(x_{i}, z_{i}, \theta\right)\right), \\
p_{\text {sum }}^{R}(x, z, t, \theta)=\sum_{i=1}^{N} p^{R}(x, z, i, t) * \delta\left(t-f\left(x_{i}, z_{i}, \theta\right)\right)
\end{array}\right.
$$

In Eq. $4, f\left(x_{i}, z_{i}, \theta\right)$ denotes the time delay imposed on the $i$-th shot wavefield when the incident angle is $\theta$. The cross-correlation imaging condition is applied to the stacked wavefields and one plane-wave image $I^{\text {plane }}$ at an angle of $\theta$ can be obtained, expressed by

$$
I^{\text {plane }}(x, z, \theta)=\sum_{t=0}^{T} p_{\text {sum }}^{S}(x, z, t, \theta) p_{\text {sum }}^{R}(x, z, t, \theta)
$$

Owing to the complexity of underground structures, obtaining a desirable migrated image from only one angle is difficult, and accomplishing multiple angles migration is necessary. Summing the plane-wave migration multi-angle images yields a final stacked image, represented by

$$
I_{\text {sum }}^{\text {plane }}(x, z)=\sum_{k_{\theta}=1}^{M} \sum_{t=0}^{T} p_{\text {sum }}^{S}(x, z, t, \theta) p_{\text {sum }}^{R}(x, z, t, \theta),
$$

where $M$ indicates the number of plane-wave angles. By comparing the conventional (Eq. 3) and plane-wave RTM (Eq. 6 ) methods, we find that the migration number of conventional RTM is the same as the number of shots $N$, while the migration number of plane-wave migration is equal to the number of plane-wave angles $M$. For PWRTM, the choice of plane-wave angles is critical, as it significantly affects the imaging accuracy and computational efficiency. It is necessary to choose a suitable number of angles to ensure balance between computational efficiency and imaging accuracy. 
To visually compare the regular and plane-wave RTM, we designed a simple velocity model with a size of $1024 \times 256$ and a grid interval of $5 \mathrm{~m}$ (Figure 2a). The sources and geophones were arranged on the surface and the seismic records were synthesized by seismic modeling using the finite difference method. We employed the first-order derivative of a Gaussian wavelet with a dominant frequency of $25 \mathrm{~Hz}$ as the source time-function. The number of shot gathers was set as 277 and shot interval as $20 \mathrm{~m}$. The recording time was set as $6 \mathrm{~s}$ and time interval as $0.4 \mathrm{~ms}$. The true velocity shown in Figure 2a was smoothed to obtain a migration velocity (Figure 2b). With the migrated velocity model and synthetic seismic records, we performed regular RTM and PWRTM to obtain the imaging results. Figure 22 illustrates the CAIGs obtained using the PWRTM (Eq. 5), which has 71 angles from $-70^{\circ}$ to $70^{\circ}$ with an interval of $2^{\circ}$. When performing the PWRTM, the maximum-delayed time was set as 2.8 $\mathrm{s}$; therefore, the total simulation time was $8.8 \mathrm{~s}$, which is approximately 1.5 times the original record length. The time delay implies an increment in computational effort. We stacked all 71 plane-wave migrated images to obtain the image shown in Figure 2e. Although this image reflects the underground structure satisfactorily and the crosstalk artifacts are well suppressed, it requires 71 migrations and is computationally inefficient. To reduce the computational burden, we selected the images corresponding to $-60^{\circ} \sim 60^{\circ}$ with an interval of $20^{\circ}$ for the CAIGs, as shown in Figure 2, and then summed them to obtain the PWRTM stacked image with seven incident angles, as shown in Figure 2 $\mathrm{g}$. It is evident that although the image in Figure $2 \mathrm{~g}$ can generally reveal the underground structures, it is heavily affected by crosstalk artifacts.

Figure $2 \mathrm{~d}$ shows the common-shot image gathers using conventional RTM (Eq. 2). For the generation of image gathers shown in Figure 2d, 277 shots correspond to 277 cycles of migration, which requires a large amount of computation time. The total images of 277 shots are stacked to obtain the image shown in Figure 2f. The imaging result is satisfactory, except for the presence of low-frequency artifacts in the shallow part. The required computation time for obtaining the results shown in Figure 2 2 -g is 4, $596 \mathrm{~s}, 29,027 \mathrm{~s}$, and $468 \mathrm{~s}$, respectively. Comparing the three images in Figure $2 \mathrm{e}-\mathrm{g}$, that in Figure $2 \mathrm{~g}$ requires the least computational consumption; however, it suffers the greatest interference from artifacts. Figure $2 \mathrm{~h}$ presents a high-resolution image derived by convolving the reflectivity of this model with a Ricker wavelet with a approximately $70 \mathrm{~Hz}$ dominant frequency. The reflectivity can be acquired from the true velocity model (Figure 2 a). The high-resolution convolved image is our expected ideal image of the subsurface structure. Based on the above findings, this study aimed to obtain high-quality imaging results with the minimum possible computation time, and deep learning provides a useful tool to help achieve this goal.

\section{Plane-WAVe RTM Image Denoising And ENHANCEMENT VIA CNN}

As mentioned earlier, plane-wave migration requires a suitable number of angles, and the same number of migrations, to achieve a balance between computational consumption and imaging quality. It is a challenge to obtain high-accuracy imaging results with a small number of migrations. In addition, Eq. 6 is a linear superposition of plane-wave migration images for multiple angles, and such a linear superposition provides limited scope for suppressing crosstalk and improving imaging quality. To resolve this limitation, we designed an end-to-end CNN architecture (Figure 3) to denoise and enhance the planewave migrated images. The proposed architecture accepts CAIGs, that is, a combination of plane-wave images with seven incident angles $\left(0^{\circ}, \pm 20^{\circ}, \pm 40^{\circ}, \pm 60^{\circ}\right)$ as input, with seven channels. The desired output of the network is a noisefree high-resolution image convolved from the corresponding reflectivity model corresponding to the Ricker wavelet with a dominant frequency of approximately $70 \mathrm{~Hz}$. The network has three potential functions: optimal stacking of the images with multiple incidence angles, crosstalk noise removal, and imaging enhancement. The proposed architecture is designed by replacing the convolutional blocks in a U-net network [48], which comprises three parts: an encoder, decoder, and refinement module [49] [50]. Regarding the encoder and decoder structure, the U-net can help extract multi-scale structural features of seismic images.

Several layers of ResNet-18 [51] are used to constitute the encoder part. ResNet-18 comprises 18 layers of residual net, and can address the degradation problem of neural networks with depth. A series of residual blocks are stacked to form the Resnet-18. Figure 4 illustrates a residual block, which comprises two $3 \times 3$ convolution filters. The shortcut of the residual block enables the network to learn the seismic structural features easily [51]. The details of the layers or blocks used in the $\mathrm{CNN}$ architecture are shown in Table I. When we input raw plane-wave CAIGs, after two $5 \times 5$ convolution layers followed by batch normalization and ReLU activation function, the feature map size becomes $1 / 2$ of the input image. After max pooling and four blocks (Block1-Block4) of Resnet-18, the feature map size successively becomes $1 / 4,1 / 8,1 / 16$, and 1/32 of the input. Here, each block among Block1-Block4 contains two residual blocks (Figure 4).

The decoder part is designed to recover the desired size of the feature map from the encoder part. We used the upsampling block with reference to [50] [52]. As shown in Figure 5, the upsampling block, which is somewhat similar to the residual block (Figure 4), has a shortcut. This upsampling structure allows for more efficient access to global information. For the upsampling block, the input feature map is first bilinearly interpolated to enlarge the size. The expanded-size feature map is then subjected to double convolutional layers and one convolutional layer, respectively. Finally, the two parts are superimposed with a shortcut. Using five upsampling blocks (UpBlock0-UpBlock4), the feature map size becomes 1/16, $1 / 8,1 / 4,1 / 2$, and 1 the size of the input images, successively. We concated the different scale layers between the encoder and decoder parts (dark blue arrows in Figure 3 .

Regarding the refinement part, we stacked 3 convolutional layers (Conv4-Conv6). Conv4 and Conv5 operate with a $5 \times 5$ convolution, batch normalization, and ReLU. The last convolutional layer (Conv6) is a straightforward $5 \times 5$ convolution 
a)

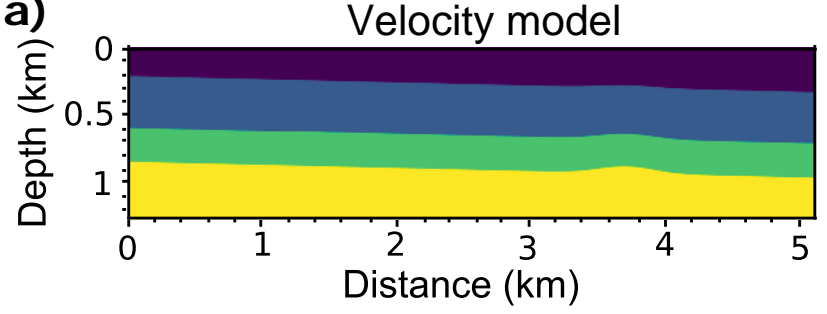

C)

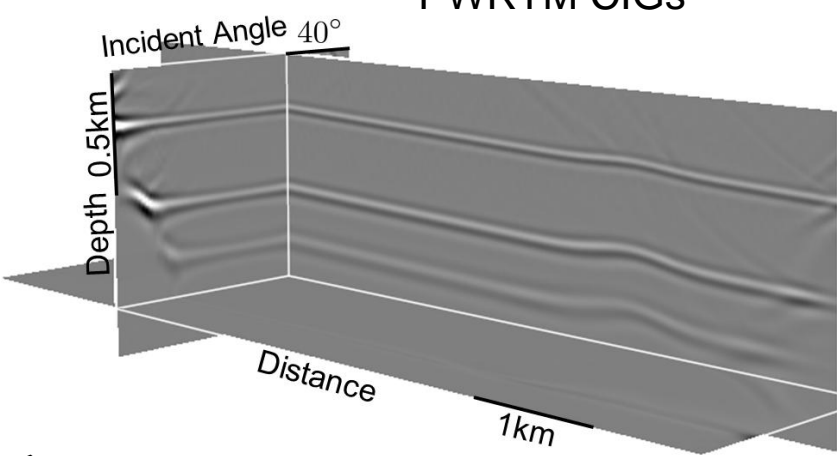

e) PWRTM stacked image with 71 angles

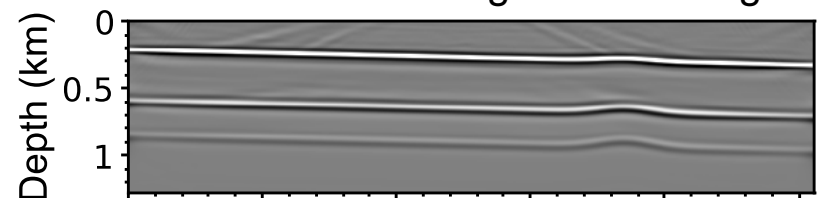

g) PWRTM stacked image with 7 angles $\mathrm{h}$

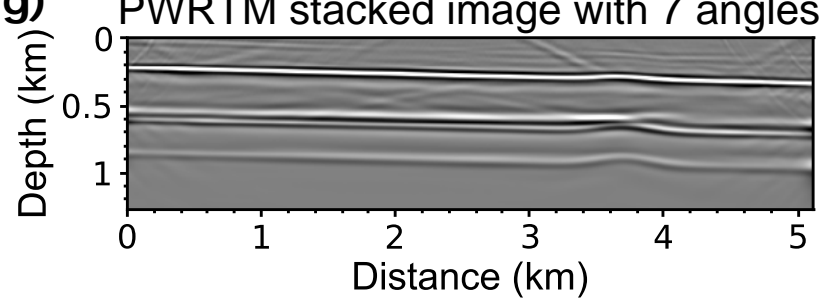

b) Migration Velocity model

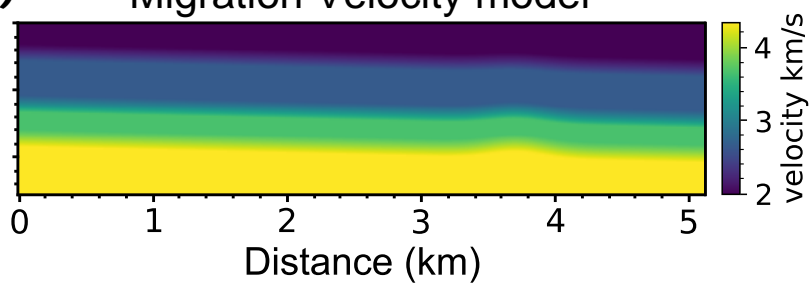

d)

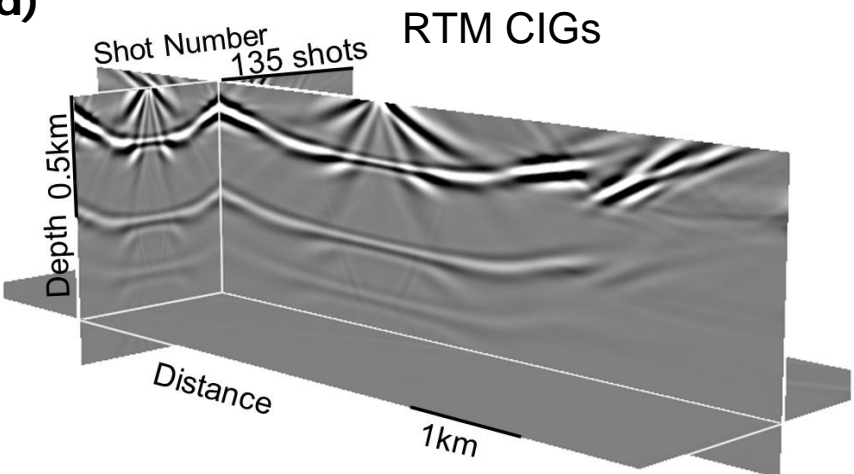

f) RTM stacked image with 277 shots

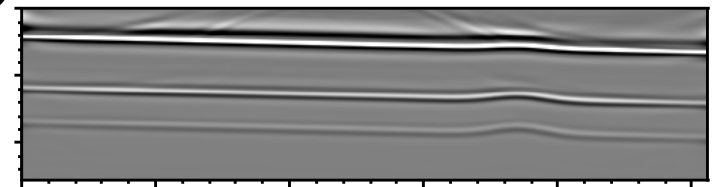

)

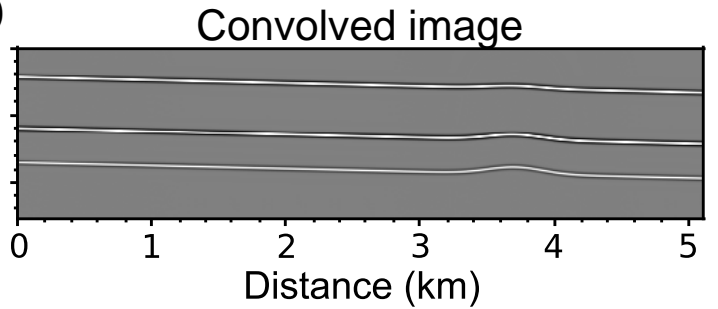

Figure 2: Four-layer velocity model and its imaging results. (a) true velocity model, (b) migration velocity model, (c) CAIGs obtained with plane-wave RTM with 71 incident angles (from $-70^{\circ} \sim 70^{\circ}$ with an interval of $2^{\circ}$ ), (d) common-shot image gathers (CSIGs) obtained using typical RTM with 277 shots, (e) PWRTM stacked image of 71 incident angles, produced by stacking the image gathers as shown in Figure 2 ; along the angular direction, (f) conventional RTM stacked image with 277 shots, (g) PWRTM stacked image of 7 incident angles $\left(-60^{\circ} \sim 60^{\circ}\right.$ with an interval of $20^{\circ}$ ), and (h) high-resolution image convolved from the corresponding reflectivity model corresponding to the Ricker wavelet with a dominant frequency of approximately $70 \mathrm{~Hz}$.

to obtain the final output of the network.

\section{A. Training datasets}

A large amount of input data and corresponding labels are essential during the training and validation of a CNN model. To implement image denoising and enhancement of planewave migration by training a $\mathrm{CNN}$, we need diverse planewave migration image gathers and corresponding targets, that is, high-resolution images convolved from the corresponding reflectivity models. By extracting multiple sub-images from a migrated image and the corresponding target image, we can obtain a large amount of training data. We designed a workflow to build velocity models and their corresponding migrated image gathers, as shown in Figure 6. In this workflow, we first created a vast number of $2 \mathrm{D}$ true velocity models of size
$1024 \times 256$. Using the velocity models, we placed sources and geophones at the surface and synthesized seismic records through finite-difference simulation. Velocity models were also employed to derive the reflectivities, which were then convolved with a Ricker wavelet with a dominant frequency of approximately $70 \mathrm{~Hz}$ to produce clear and high-resolution convolved images. The true velocities were smoothed and were used for the plane-wave migration to obtain the imaging gathers. At each iteration during training, the image gathers were fed into the network, producing predicted images. We calculated the residuals between the predicted images and the corresponding clear images (i.e., the high-resolution convolved images), and we back-propagated the residuals to the network. In training, the network adjusts the parameters until they meet our requirements. 


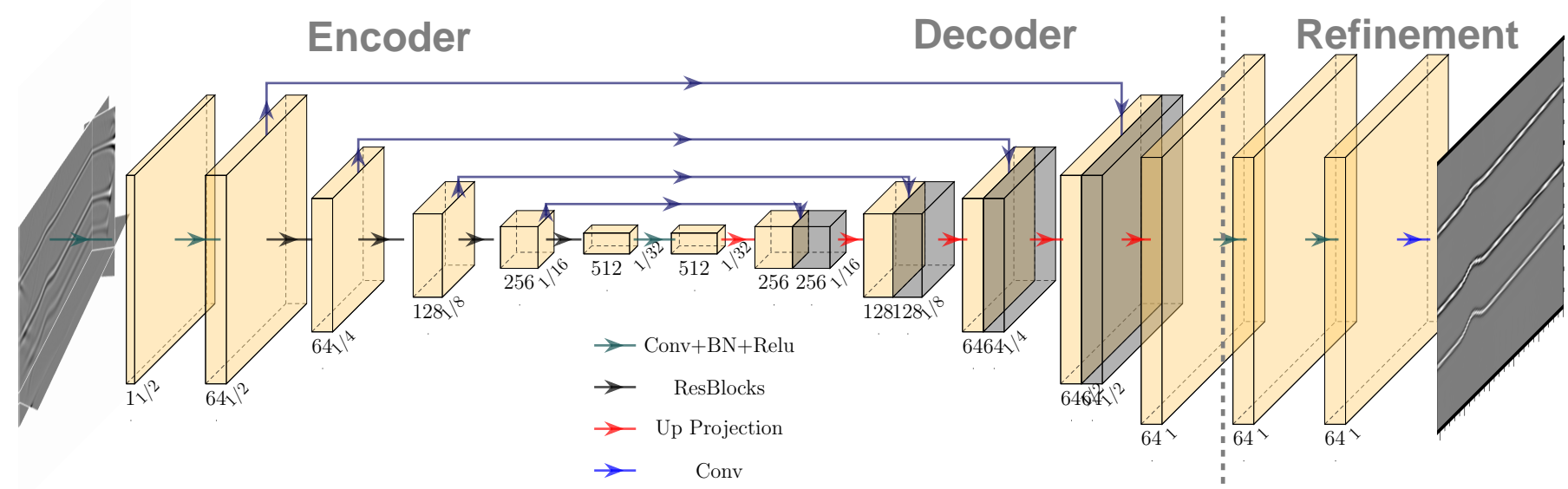

Figure 3: Proposed CNN architecture. The input is noisy CAIGs of PWRTM, which is a combination of plane-wave images with seven incident angles $\left(0^{\circ}, \pm 20^{\circ}, \pm 40^{\circ}\right.$, and $\left.\pm 60^{\circ}\right)$ and the output is a clear and enhanced image. The CNN architecture comprises encoder, decoder, and refinement parts. The yellow rectangles are the layers inside the CNN. The size of these rectangles denotes the size of each layer's features map and the numbers below them denote the number of channels for each layer. The arrows indicate the operations between different layers.

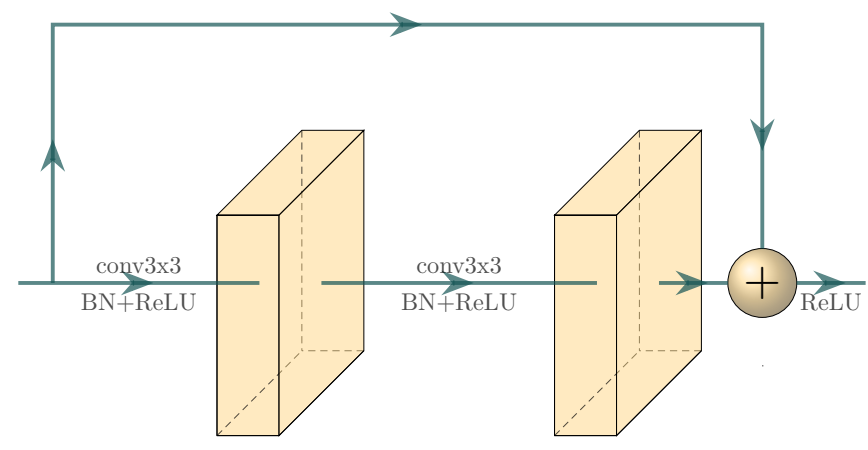

Figure 4: Structure of residual block.

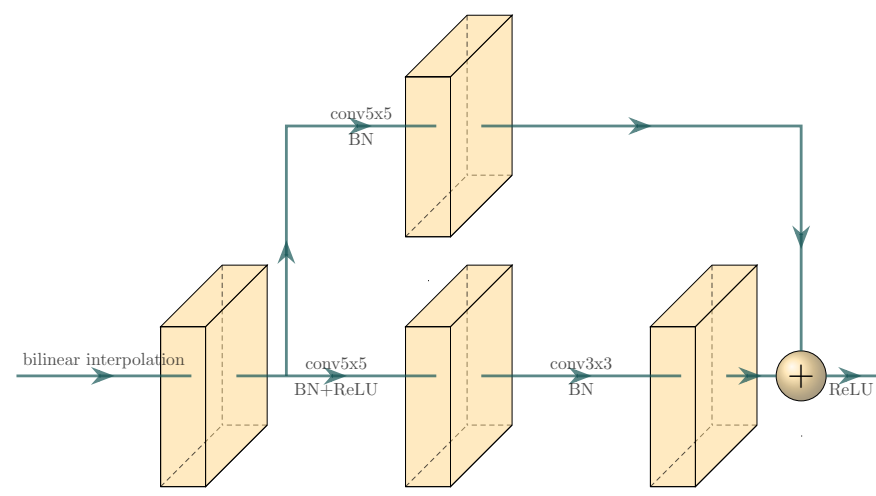

Figure 5: Structure of upsampling block.

1) Synthetic velocity models and seismic records: As shown in the workflow in Figure 6, the formation of image gathers and labels requires velocity models and seismic records. The

\begin{tabular}{cccccc}
\hline Section & Layer/Block & $\begin{array}{c}\text { Kernel } \\
\text { Size }\end{array}$ & Scale & $\begin{array}{c}\text { Input } \\
\text { Channel }\end{array}$ & $\begin{array}{c}\text { Output } \\
\text { Channel }\end{array}$ \\
\hline \multirow{5}{*}{ Encoder } & Conv1 & 5 & $1 / 2$ & 7 & 7 \\
& Conv2 & 5 & $1 / 2$ & 7 & 64 \\
& Pool1 & 3 & $1 / 4$ & 64 & 64 \\
& Block1 & & $1 / 4$ & 64 & 64 \\
& Block2 & & $1 / 8$ & 64 & 128 \\
& Block3 & & $1 / 16$ & 128 & 256 \\
& Block4 & & $1 / 32$ & 256 & 512 \\
\hline \multirow{5}{*}{ Decoder } & Conv3 & 1 & $1 / 32$ & 512 & 512 \\
& UpBlock0 & & $1 / 16$ & 512 & 256 \\
& UpBlock1 & & $1 / 8$ & 512 & 128 \\
& UpBlock2 & & $1 / 4$ & 256 & 64 \\
& UpBlock3 & & $1 / 2$ & 128 & 64 \\
& UpBlock4 & & 1 & 128 & 64 \\
\hline \multirow{5}{*}{ Refinement } & Conv4 & 5 & 1 & 64 & 64 \\
& Conv5 & 5 & 1 & 64 & 64 \\
& Conv6 & 5 & 1 & 64 & 64 \\
\hline
\end{tabular}

Table I: Details of the layers or blocks used in each section of the CNN architecture, including kernel size, scale, and the number of channels of the input and output feature maps.

structural diversity of velocity models has a strong impact on the quality of output images. We used two schemes to automatically build different types of velocities. For the first scheme, we followed the work of [53] to create the velocity models. We started with three dimensional (3D) flat velocity models (Figure $7 \mathrm{k}$ ), and then added the folding (Figure $7 \mathrm{~b}$ ), and faulting (Figure 77). Thus, we constructed 100 3D velocity models embodying different structures. We extracted one vertical slice from these $3 \mathrm{D}$ velocity models along the inline direction and obtained 100 2D-velocity models.

In the second scheme, we performed random extractions from the Marmousi velocity model [54] (Figure 8) and conducted affine transformations on these extractions using the 


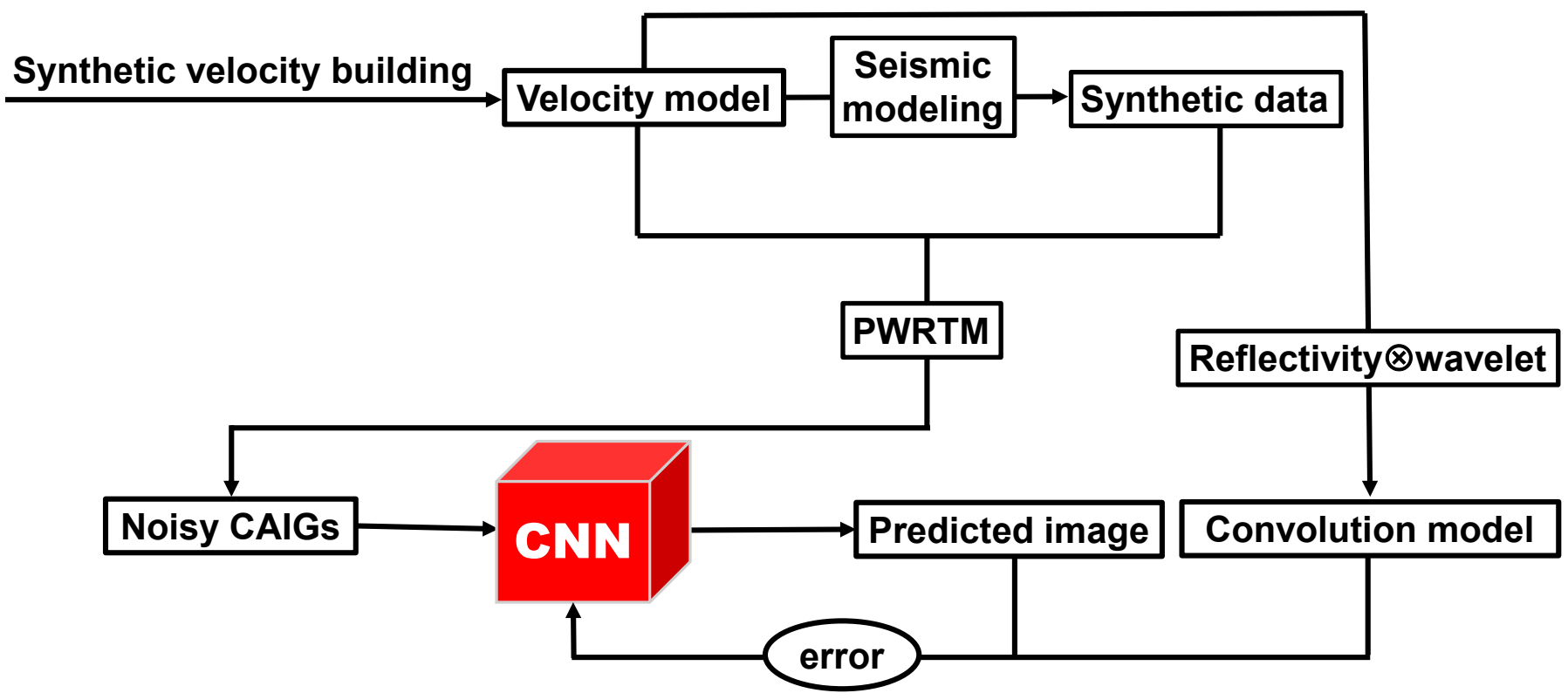

Figure 6: Workflow for building training datasets.
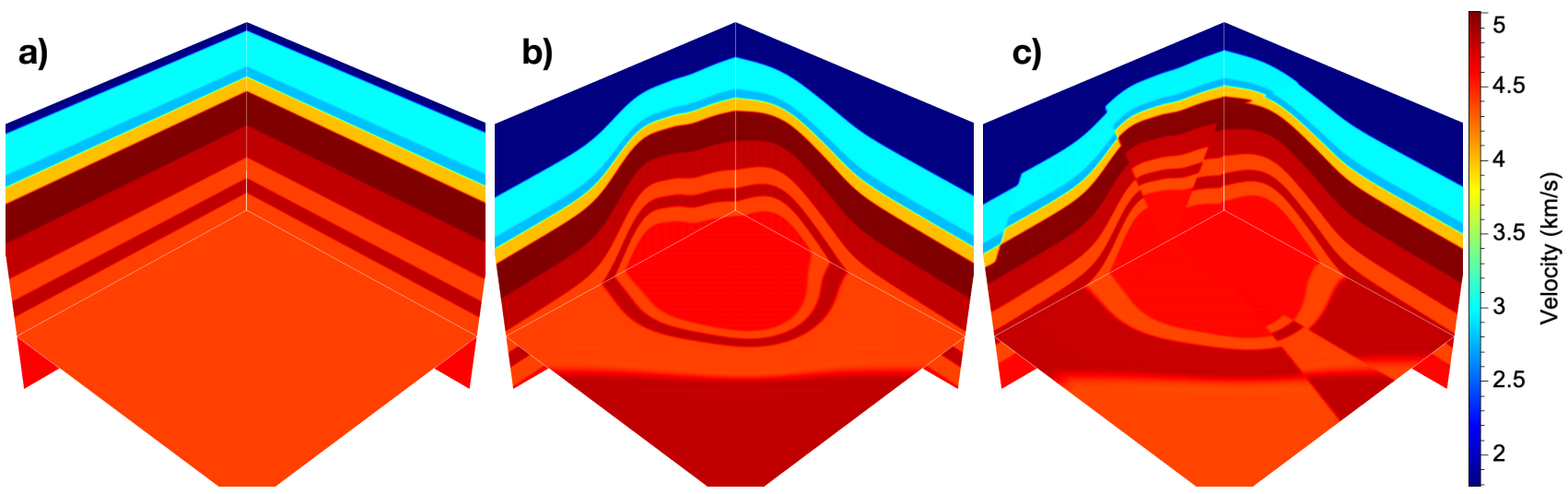

Figure 7: Construction of 3D velocity models. (a) A flat model, (b) folded model, and (c) fault model.

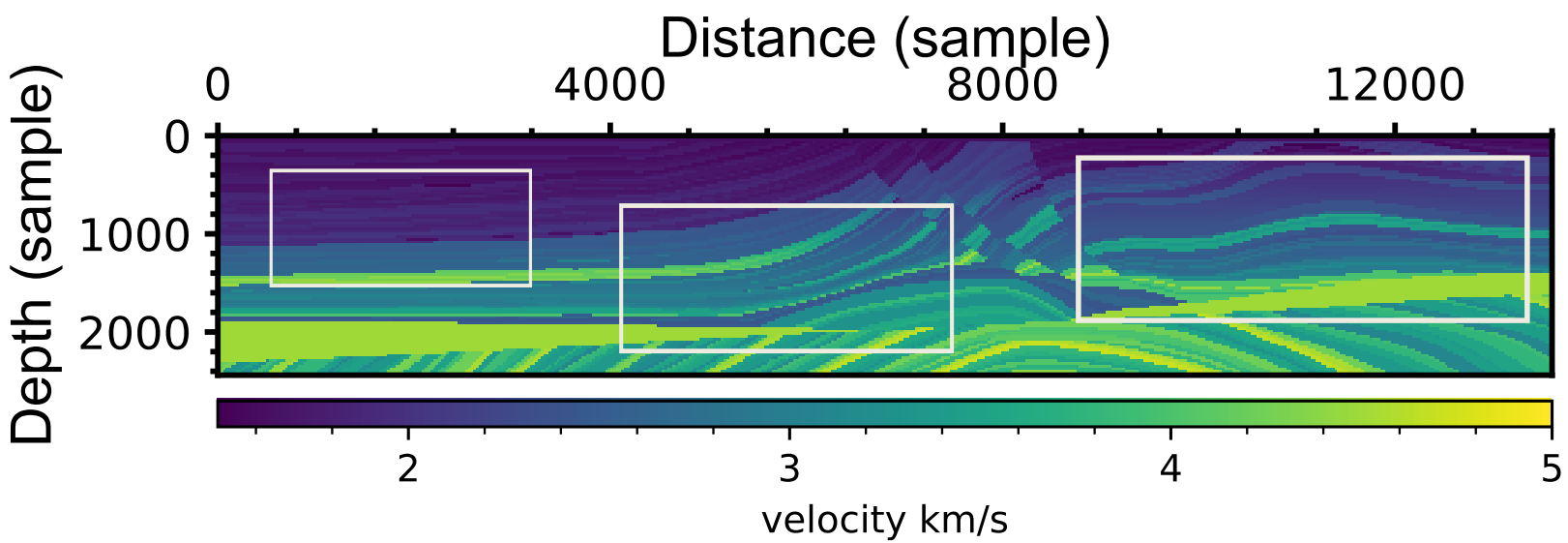

Figure 8: Marmousi velocity model. To create the velocity models, we randomly extracted different regions (white rectangle boxes) of the model and applied affine transformations on the extractions to obtain diverse structural velocities.

augmentor tool [55]. The affine transformations include elastic distortions, perspective transforms, zooms, and their combinations. 405 velocity models were created. Notably, although the sizes of the randomly chosen regions were not fixed, they had the same sampling number of 1024 (distance) $\times 256$ (depth).

Figure 9 shows six typical velocity models, and we observed that these velocity models contain either simple or complex structures, such as horizontal layers, folds, and faults. The richness of models can help the proposed CNN perform well at learning the image features. Using the velocity models, we simulated the corresponding seismic records. The simulations were performed with four dominant frequencies of the source 


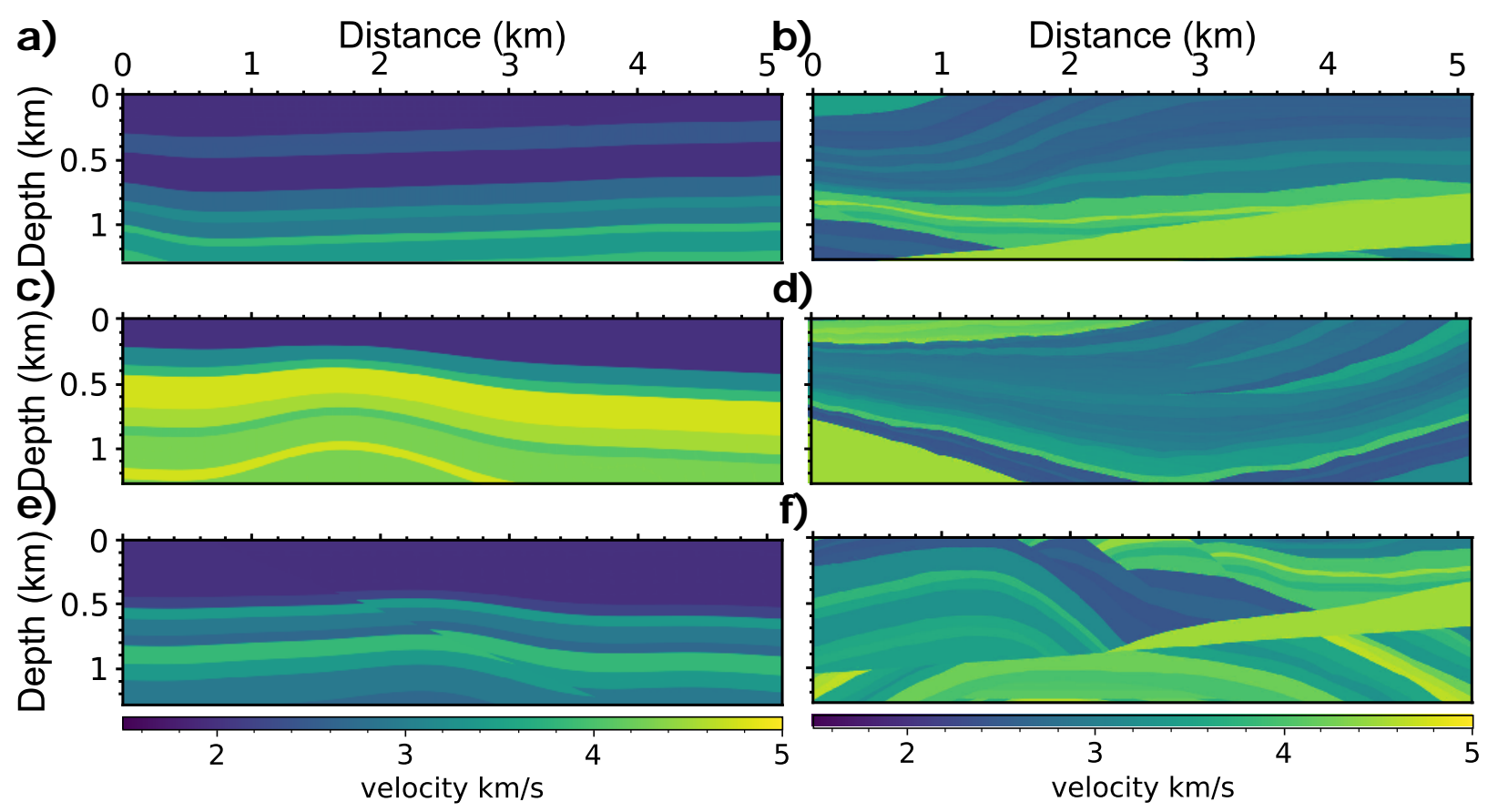

Figure 9: Velocity models with different structures.

wavelets: $15 \mathrm{~Hz}, 20 \mathrm{~Hz}, 25 \mathrm{~Hz}$, and $30 \mathrm{~Hz}$. The sources and geophones were distributed on the entire surface. The number of shots was 277 .

2) Synthetic migrated images and high-resolution images convolved from the corresponding reflectivity models: Using the velocity models and corresponding synthetic seismic records, we can employ plane-wave RTM to obtain migrated image gathers. The smoothed velocities were used for the plane-wave RTM (Eq. 5), forming CAIGs (Figure 10). We can observe that these images are interrupted by crosstalk artifacts, which even obscure the true signals.

The true velocities were employed to obtain high-resolution images convolved from the corresponding reflectivity models (Figure 11). Compared to the noisy image gathers (Figure 10, the 'true' images in Figure 11 are clear and present the underground structure accurately. In terms of computational efficiency, the noisy image gathers shown in Figure 10 require only seven cycles of migration because of the implementation of simplified PWRTM with seven angles to generate the raw images.

\section{B. Loss functions}

We defined a loss function based on the Laplacian pyramid$\mathcal{L} 1$ loss to train and validate the proposed network. The loss function follows the form presented in [56]

$$
\mathcal{L}_{\text {lap }}=\sum_{j}^{M} 2^{2 j} \mid\left(L^{j}\left(y_{k}^{p}\right)-L^{j}\left(y_{k}\right) \mid,\right.
$$

where $L^{j}\left(y_{k}^{p}\right)$ and $L^{j}\left(y_{k}\right)$ represent the $j$-th level Laplacian pyramid features of the predicted and label images, respectively. In our training process, $M$ equaled 5 , which means that $\mathcal{L} 1$ loss function was conducted on images at five different scales. The image features at different scales were produced by average pooling. The Laplacian pyramid loss is of potential importance in multi-scale image denoising and enhancement.

\section{Training and Validation}

Utilizing the above-discussed method, we synthesized a total of 505 datasets. We utilized approximately $80 \%$ of the datasets for training and the remaining for validation. To expand the training data, four randomly extracted data of size $512 \times 128$ were trained for each epoch of the dataset during training and validation of the network. We normalized the input image gathers along channel directions to ensure their amplitude consistency.

We used the Pytorch version 1.0 [57] for the denoising and enhancement of seismic images. The network was trained using NVIDIA Tesla V100 GPU with 32 GB memory. The model parameters were randomly initialized before model training. The batch size was set as 32 , with an initial learning rate of $10^{-4}$.

We performed training with the Adam optimizer [58] on these hyperparameters and normalized seismic image gathers to update the model parameters. We terminated the training process after 300 epochs of training and validation. Figure 12 shows the loss of the trained model, and it is visible that both the training and valid losses converge to small values.

\section{Numerical Examples}

To test the trained model, we designed velocity models with different structures, as shown in Figure 13. These velocity models contained horizontal layers, folds, faults, and their combinations. The sampling size of the velocity models was $1024 \times 256$. With these velocity models, we employed the previously mentioned approach of building training datasets to obtain the corresponding plane-wave image gathers and high-resolution images. For plane-wave migration, different dominant frequencies of seismic wavelets were used to produce images with varying resolutions. Allowing for responses to different frequencies, the models shown in Figure 13 
a)

C)

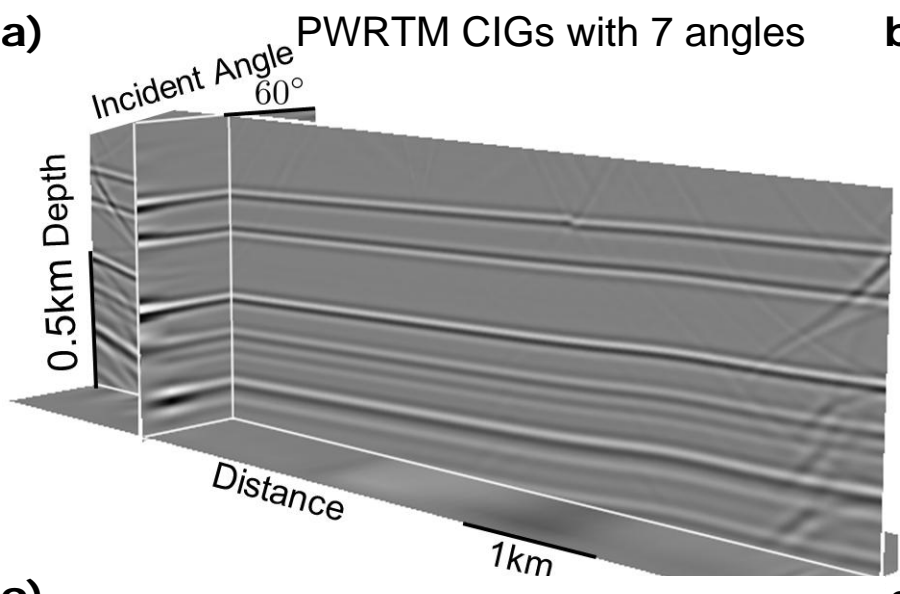

e)
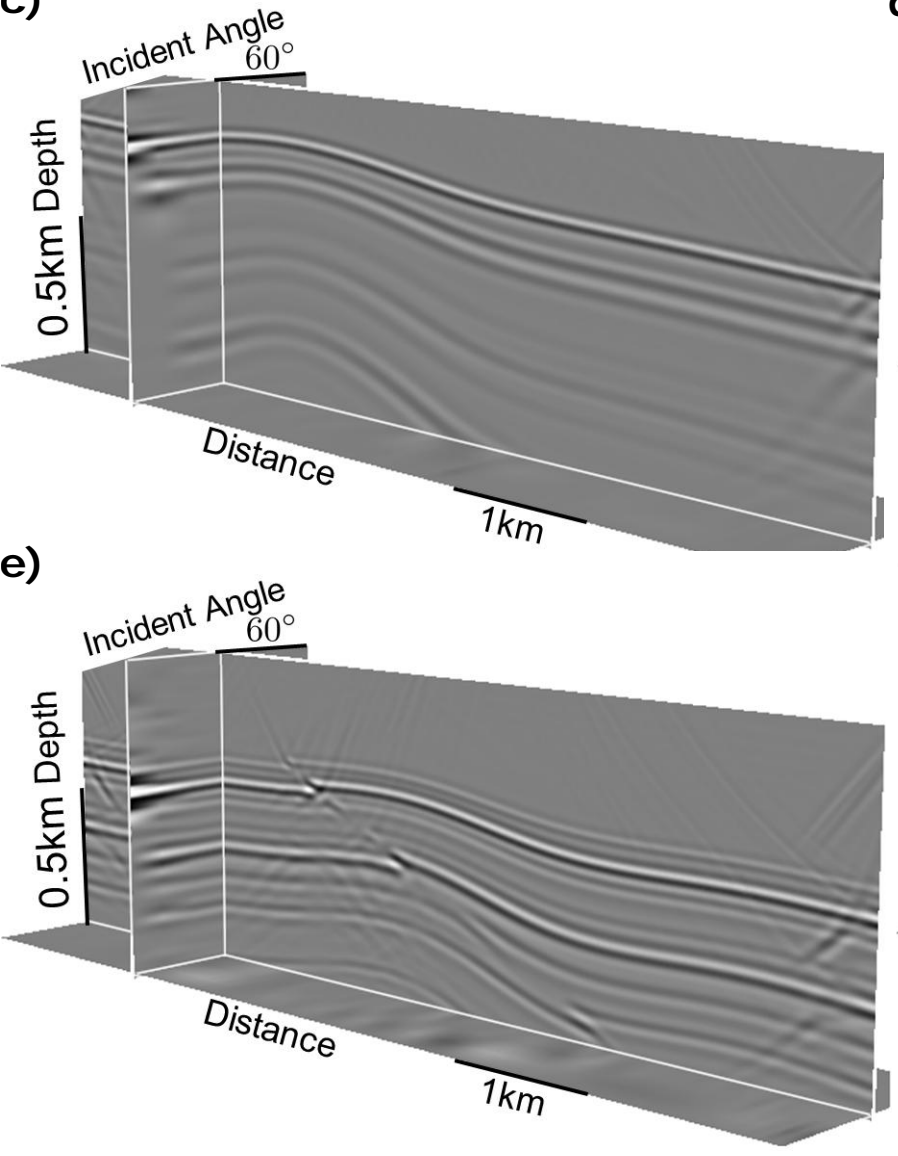

b)

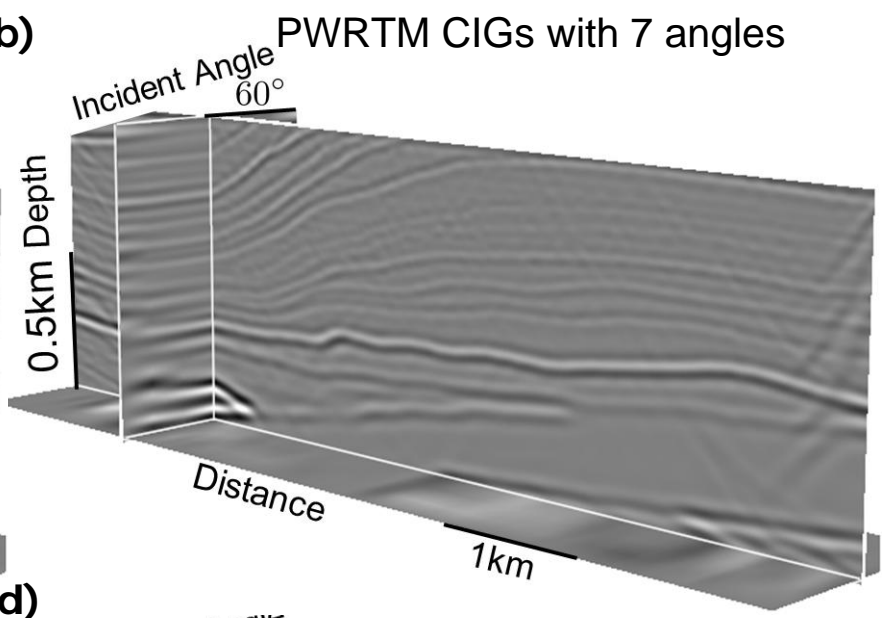

d)

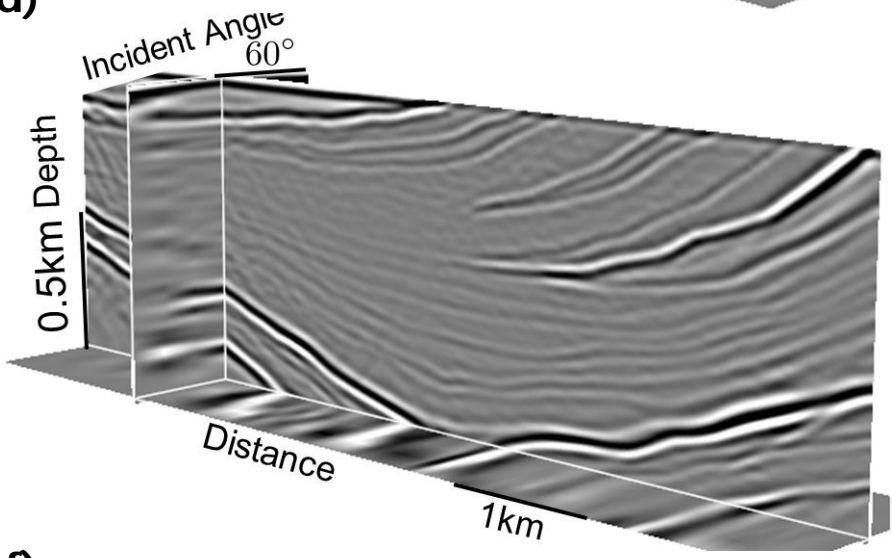

f)

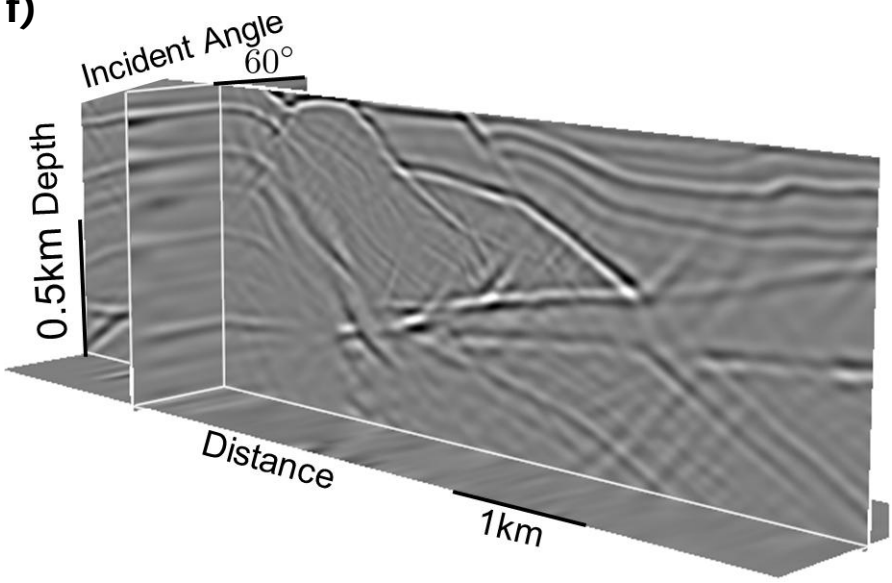

Figure 10: Plane-wave image gathers corresponding to the velocity models shown in Figure 9 . These imaging gathers have seven incidence angles $\left(0^{\circ}, \pm 20^{\circ}, \pm 40^{\circ}, \pm 60^{\circ}\right)$ and are heavily disturbed by crosstalks.

were setup with different dominant frequencies of wavelets, including $20 \mathrm{~Hz}, 25 \mathrm{~Hz}, 25 \mathrm{~Hz}, 25 \mathrm{~Hz}, 30 \mathrm{~Hz}$, and $30 \mathrm{~Hz}$.

To demonstrate the effectiveness of our method, we compared three types of images: stacked PWRTM image (i.e., stack of the input images), predicted image, and ground truth. The imaging results corresponding to the velocity models shown in Figure 13 are illustrated in Figure 14. The planewave stacked images are displayed in the first column in Figure 14, and severe interference from crosstalk noise can be observed, with low resolution and poor imaging quality. Similar to the ground truths (third column in Figure 14), the predicted images (second column in Figure 14) are free from interference from crosstalk artifacts and the signals are enhanced. In addition, the predicted images have high resolution and imaging quality. Owing to the frequency-band limitation of the wavelets and the effect of crosstalk noises, thin layers are difficult to recognize in the plane-wave stacked images, whereas the same are clearly visible in the high-resolution predicted images (marked by red arrows in Figure 14p. Hence, the designed network achieves optimal superposition of imaging gathers, suppression of crosstalk artifacts, and imaging enhancement.

Next, used the training network to test a model with greater complexity, as illustrated in Figure 15 Figure 15 a shows the 


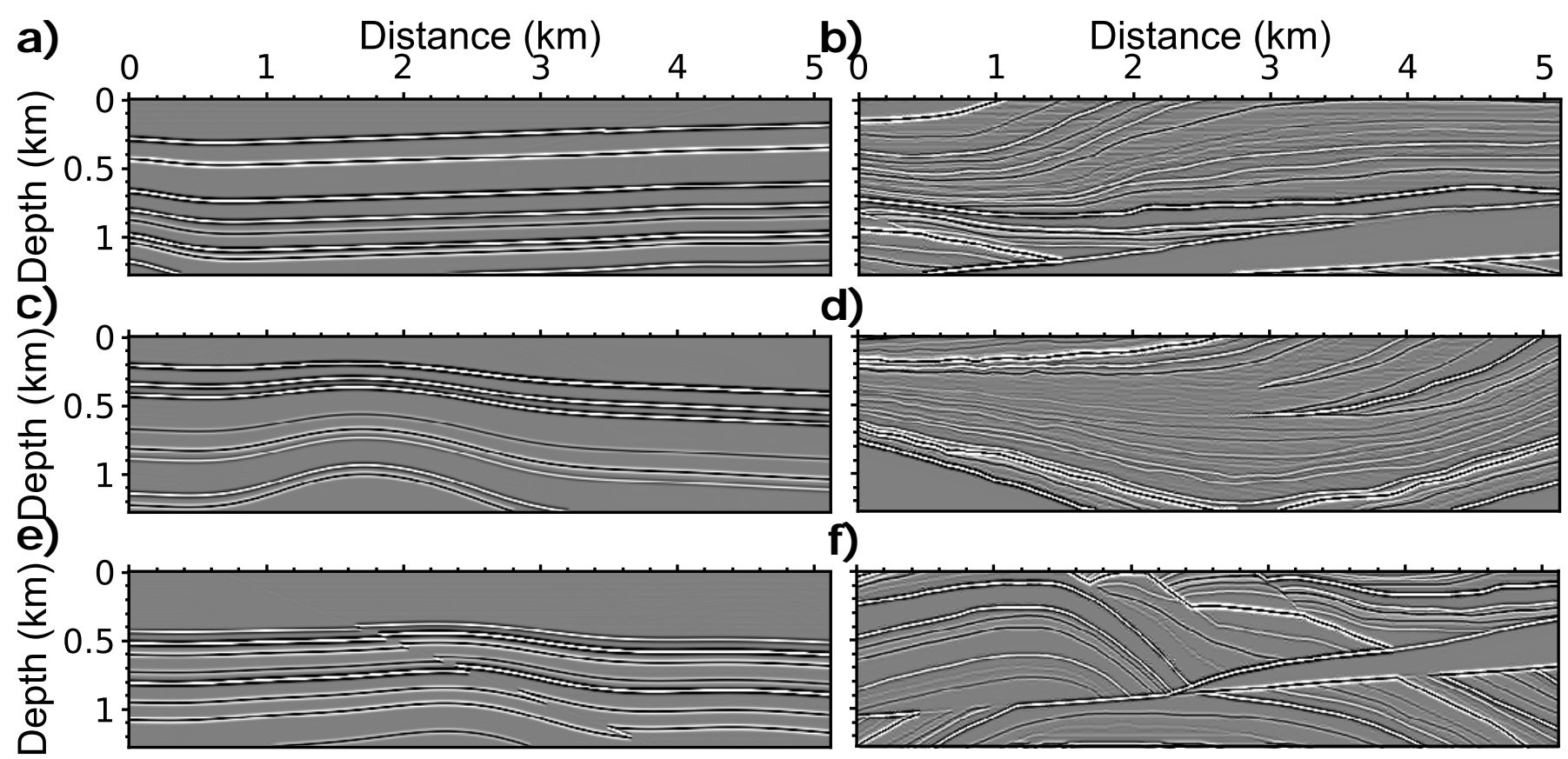

Figure 11: High-resolution images convolved from the corresponding reflectivity models corresponding to the velocities depicted in Figure 9. These images have high quality and are free from crosstalk artifacts, as desired for subsurface images.

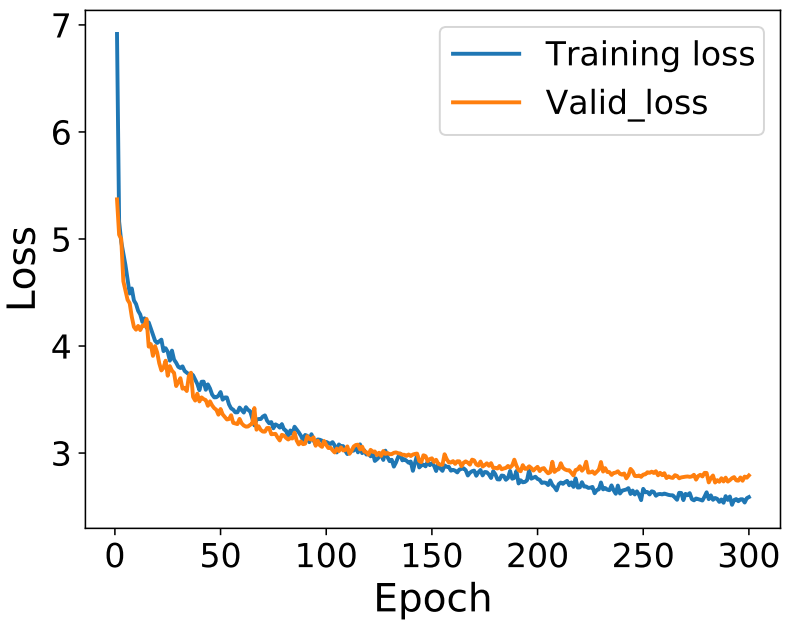

Figure 12: Loss of designed network for training and validation datasets.

velocity model with a size of $1024 \times 256$, with dense layers and steeply dipping folds and multiple faults. This complex model tends to present difficulties in seismic imaging. The parameters are similar to the model shown in Figure 2 except that the delay time is $3.6 \mathrm{~s}$. To demonstrate the merits of the trained model, we compared the predicted image (Figure 15 ) with the high-resolution image (Figure $15 \mathrm{~b}$ ) convolved from the corresponding reflectivity model, PWRTM stacked image (Figure 15k), traditional RTM stacked image (Figure 15d), and stacked image of input image gathers (Figure 15). The PWRTM stacked image shown in Figure 15 c was produced by summing plane-wave images at 71 angles (from $-70^{\circ} \sim 70^{\circ}$ with an interval of $2^{\circ}$ ), which requires 71 migrations. The RTM stacked image exhibited in Figure $15 \mathrm{~d}$ was generated by summing single-shot images of 277 shots, which requires 277 migrations. Both these types of stacked images (Figure 15: and d) allow for clear imaging of subsurface structures without crosstalk artifacts. However, they are limited in resolution and insensitive to fine layers owing to the influence of wavelet frequencies (red arrows in Figure 15 and d). The plane-wave CAIGs of seven angles (from $-60^{\circ} \sim 60^{\circ}$ with an interval of $20^{\circ}$ ) were fed into the network to obtain the predicted image shown in (Figure 15). The stacked image of the seven input image gathers is shown in Figure 15e. The image in Figure 15e is heavily affected by crosstalk noise and has poor imaging quality. Similar to the ground truth depicted in Figure 15b, the predicted image (Figure 15) eliminates crosstalk artifacts from the input image gathers and improves the imaging resolution for high-quality imaging of subsurface fine structures.

The processing time required to achieve the imaging results illustrated in Figure 15 is presented in Table II] The RTM stacked image with 277 shots required $28,790 \mathrm{~s}$, and the PWRTM stacked image with 71 angles required 4,602 s, which is approximately $16 \%$ of the time required for the traditional RTM. The processing time required for the two stacked images is large and unacceptable. In comparison, the proposed method takes only $470 \mathrm{~s}$, which is approximately $1.6 \%$ and $10 \%$ of the time required for the traditional and plane-wave RTM, respectively. The extremely high computational efficiency of our method is because of the lower number of migrations. The proposed method, involving only seven migrations, achieves both crosstalk denoising and imaging enhancement through optimal superposition of imaging gathers and maintains high computational efficiency. The proposed method breaks through the contradiction between computational efficiency and imaging accuracy to obtain high-quality imaging results with low computational cost. The predicted imaging results enable the characterization of fine subsurface 


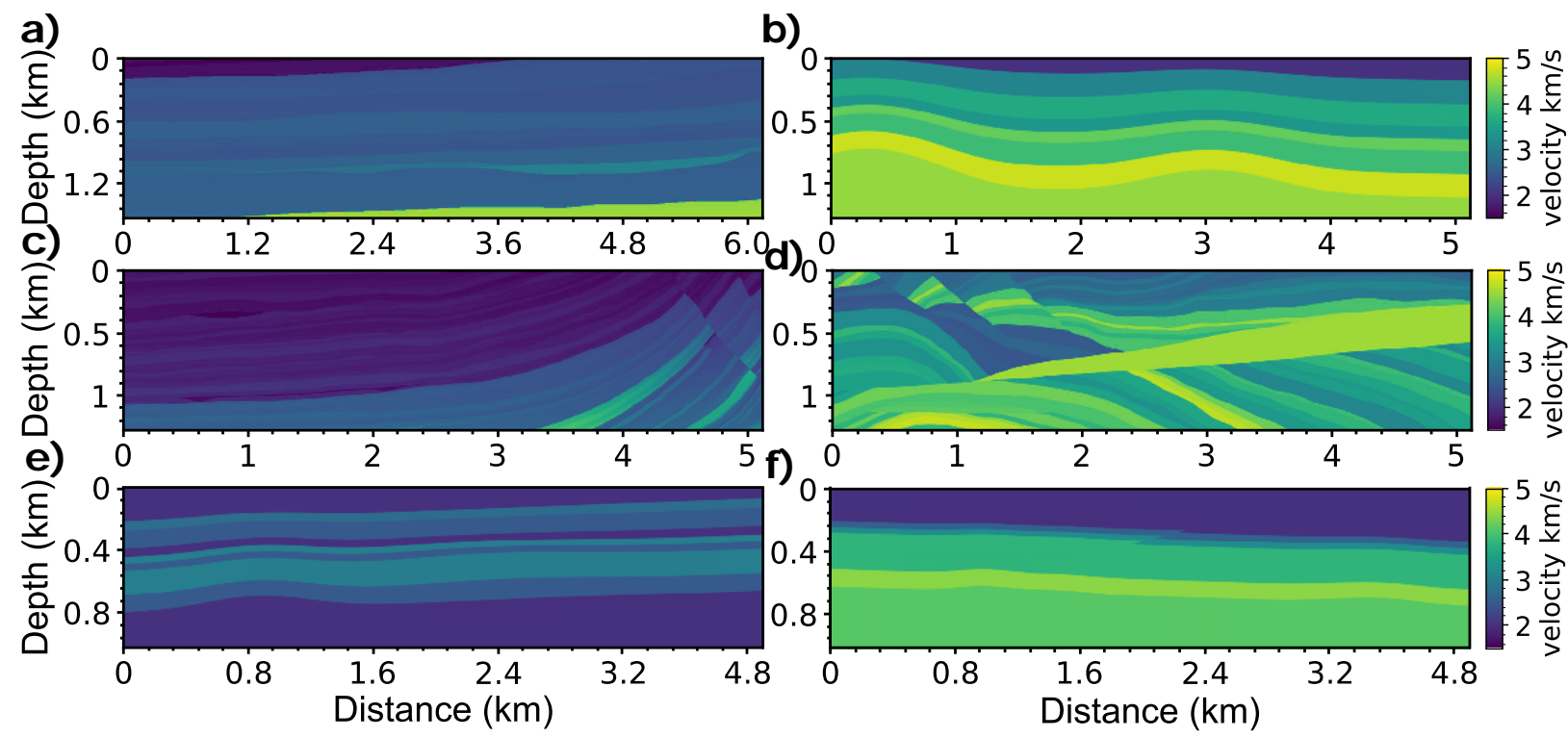

Figure 13: Velocity models with different structures for testing the trained model.

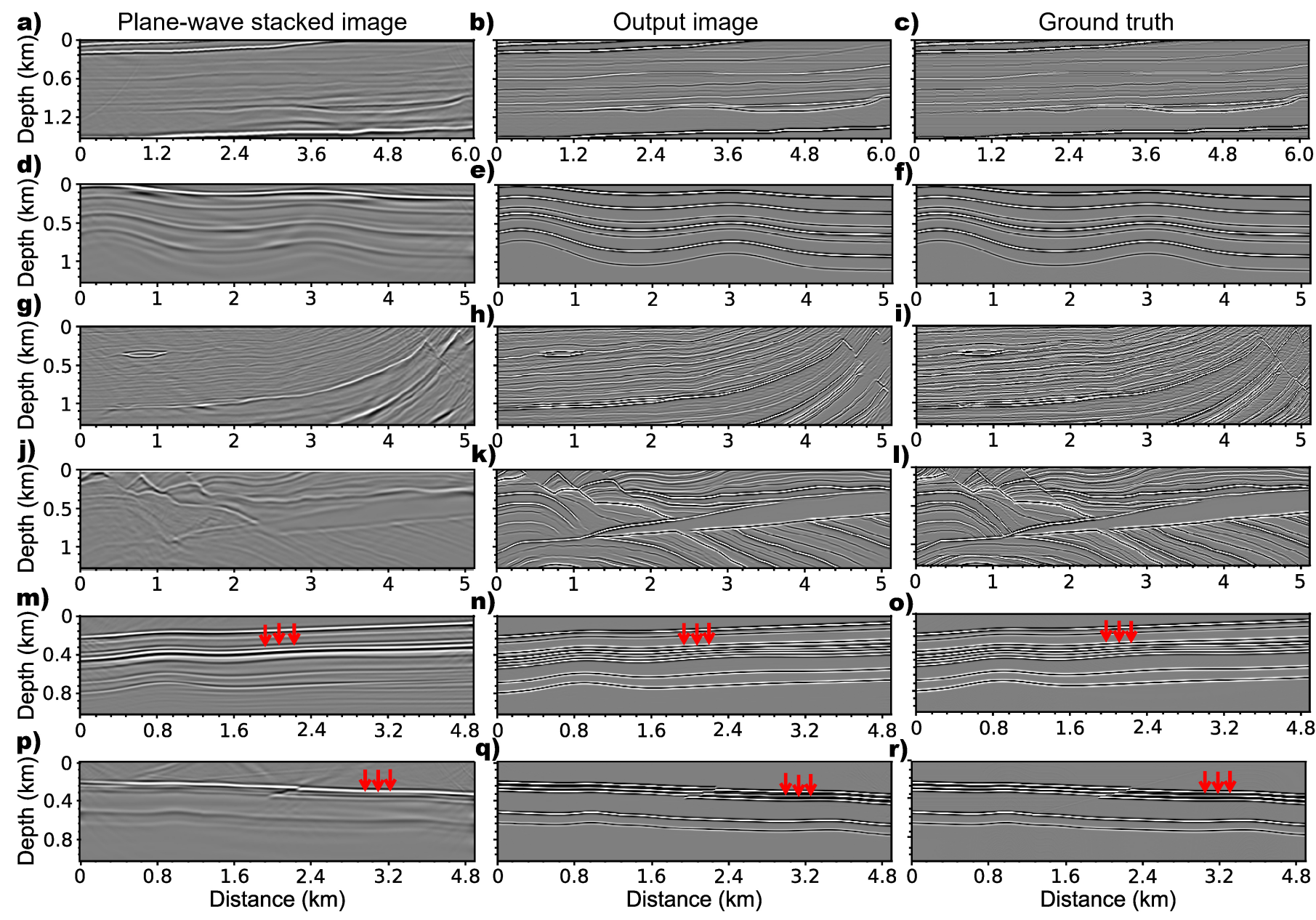

Figure 14: Imaging results corresponding to the velocities depicted in Figure 13 The images in the first column are the planewave stacked images, which are the summation of input image gathers. The second column shows the output of the network. The images in the third column are ground truths (high-resolution images convolved from the corresponding reflectivity models).

structures.

IV. CONCLUSiOnS

In this study, we presented a CNN for enhancing PWRTM. The network performs an optimal stacking of input noisy 

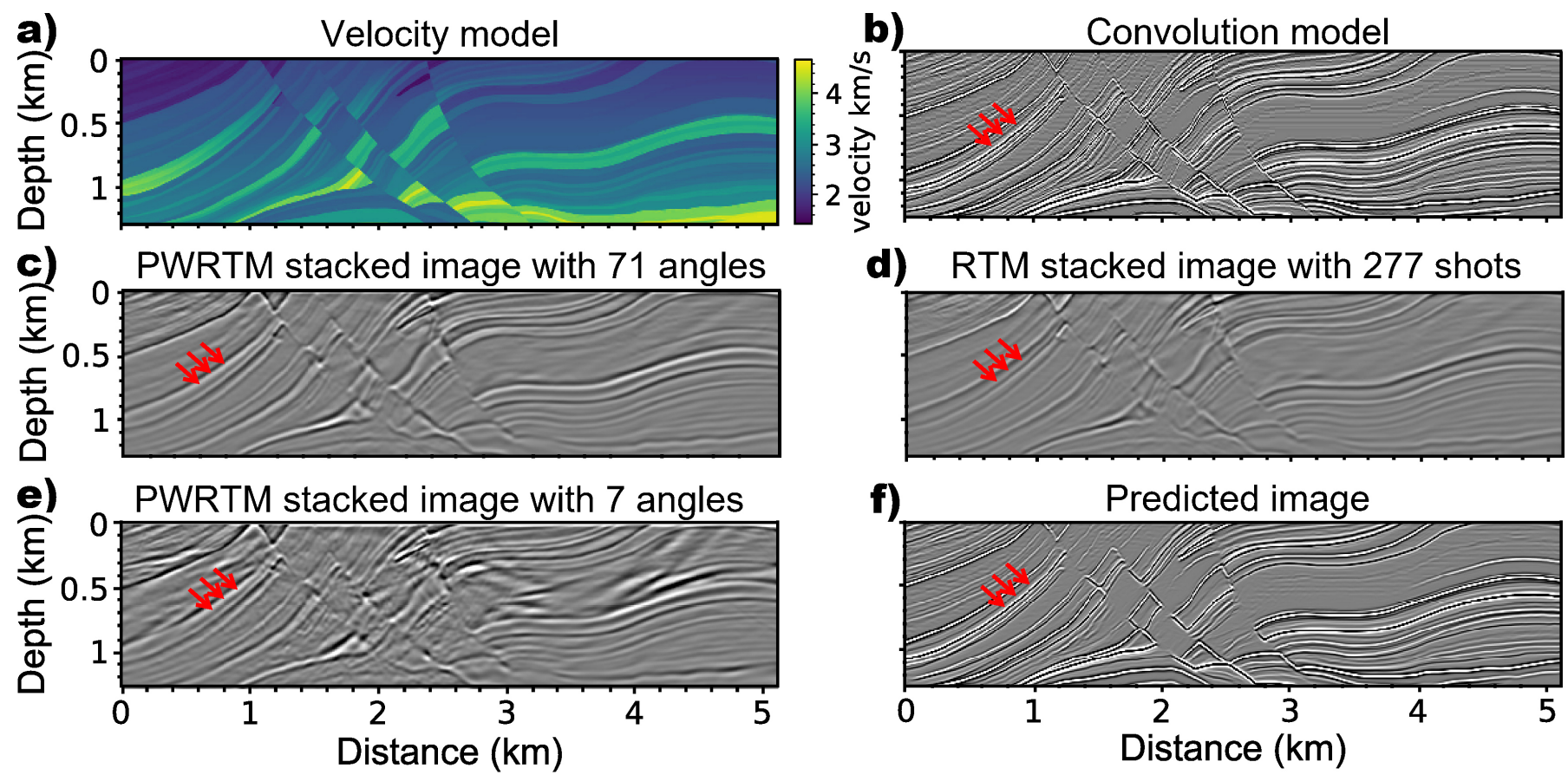

Figure 15: Velocity model with complex structures and its imaging results. (a) True velocity model, (b) high-resolution image convolved from the corresponding reflectivity model using Ricker wavelet with a dominant frequency of approximately $70 \mathrm{~Hz}$, (c) PWRTM stacked image with 77 incident angles (from $-70^{\circ} \sim 70^{\circ}$ with an interval of $2^{\circ}$ ), (d) RTM stacked image with 277 shots, (e) PWRTM stacked image with 7 incident angles $\left(-60^{\circ} \sim 60^{\circ}\right.$ with an interval of $20^{\circ}$ ), and (f) predicted image.

\begin{tabular}{ccccc}
\hline Methods & $\begin{array}{c}\text { RTM } \\
\text { with 277 shots }\end{array}$ & $\begin{array}{c}\text { PWRTM } \\
\text { with 71 angles }\end{array}$ & $\begin{array}{c}\text { PWRTM } \\
\text { with 7 angles }\end{array}$ & Our method \\
\hline $\begin{array}{c}\text { Processing time } \\
\text { (second) }\end{array}$ & 28,790 & 4,602 & 469 & $\mathbf{4 7 0}$ \\
\hline
\end{tabular}

Table II: Processing time required for the different imaging results presented in Figure 15.

plane-wave CAIGs and predicts a noise-free and high-quality image. To train the network, we designed a workflow to build numerous training datasets with diverse structures. At each iteration of the training and validation process, random extraction of migration images helped expand the dataset. A Laplacian pyramid loss function was defined to train the network architecture. The results of training and validation experiments demonstrate that our trained $\mathrm{CNN}$ is effective in eliminating the artifacts and improving the imaging quality of plane-wave CAIGs. Compared with the traditional RTM and the conventional use of several angles for plane-wave migration, the proposed method requires image gathers with only seven incident angles, improving both computational efficiency and imaging quality. The predicted images have higher resolution and can clearly present the fine subsurface structures. In terms of time consumption, the proposed method requires approximately $1.6 \%$ and $10 \%$ of the time required by RTM and PWRTM, respectively, for processing a provided test model with a size of $1024 \times 256$. Extending the image denoising and enhancing tasks for $2 \mathrm{D}$ plane-wave migration to $3 \mathrm{D}$ migration is straightforward by directly replacing the $2 \mathrm{D}$ convolutional kernel with a 3D kernel. However, building the training datasets requires a relatively large amount of computation compared with the 2D case. The method described in this

study has the potential for generalization to other geophysical imaging methods.

\section{ACKNOWLEDGMENTS}

The authors acknowledge the critical comments received from Dr. Yunzhi Shi, Wenyang Chen, Bin Chen, and Jintao Li. This study received support from the National Natural Science Foundation of China (No. 41774121 \& 42074125) and the Joint Open Fund of Mengcheng National Geophysical Observatory (No. MENGO-202005).

\section{REFERENCES}

[1] G. Cambois, S. Ronen, and X. Zhu, "Wide-azimuth acquisition: True 3d at last!," The Leading Edge, vol. 21, no. 8, pp. 763-763, 2002.

[2] N. Moldoveanu, J. Kapoor, and M. Egan, "Full-azimuth imaging using circular geometry acquisition," The Leading Edge, vol. 27, no. 7, pp. 908-913, 2008.

[3] S. H. Gray, Seismic, Migration, pp. 1236-1244. Dordrecht: Springer Netherlands, 2011.

[4] J. B. Bednar, "A brief history of seismic migration," Geophysics, vol. 70, no. 3, pp. 3MJ-20MJ, 2005.

[5] J. Etgen, S. H. Gray, and Y. Zhang, "An overview of depth imaging in exploration geophysics," Geophysics, vol. 74, no. 6, pp. WCA5WCA17, 2009.

[6] W. S. French, "Computer migration of oblique seismic reflection profiles," Geophysics, vol. 40, no. 6, pp. 961-980, 1975.

[7] W. A. Schneider, "Integral formulation for migration in two and three dimensions," Geophysics, vol. 43, no. 1, pp. 49-76, 1978.

[8] B. Biondi, "Kirchhoff imaging beyond aliasing," Geophysics, vol. 66, no. 2, pp. 654-666, 2001.

[9] N. R. Hill, "Prestack gaussian-beam depth migration," Geophysics, vol. 66, no. 4, pp. 1240-1250, 2001.

[10] Y. Sun, F. Qin, S. Checkles, and J. P. Leveille, "3-d prestack kirchhoff beam migration for depth imaging," Geophysics, vol. 65, no. 5, pp. $1592-1603,2000$ 
[11] S. Jin, R.-S. Wu, and C. Peng, "Seismic depth migration with pseudoscreen propagator," Computational Geosciences, vol. 3, no. 3, pp. 321$335,1999$.

[12] J. m. H. Le Rousseau and M. V. de Hoop, "Modeling and imaging with the scalar generalized-screen algorithms in isotropic media," Geophysics, vol. 66, no. 5, pp. 1551-1568, 2001.

[13] E. Baysal, D. D. Kosloff, and J. W. Sherwood, "Reverse time migration," Geophysics, vol. 48, no. 11, pp. 1514-1524, 1983.

[14] H.-W. Zhou, H. Hu, Z. Zou, Y. Wo, and O. Youn, "Reverse time migration: A prospect of seismic imaging methodology," Earth-science reviews, vol. 179, pp. 207-227, 2018.

[15] W. Dai and G. T. Schuster, "Plane-wave least-squares reverse-time migration," Geophysics, vol. 78, no. 4, pp. S165-S177, 2013.

[16] J. Godwin and P. Sava, "A comparison of shot-encoding schemes for wave-equation migration," Geophysical Prospecting, vol. 61, pp. 391408, 2013.

[17] J. Hu, H. Wang, L. Zhao, Y. Shao, M. Wang, and A. Osen, "Efficient reverse time migration with amplitude encoding," Journal of Geophysics and Engineering, vol. 12, no. 4, pp. 601-609, 2015.

[18] F. Perrone and P. Sava, "Wave-equation migration with dithered plane waves," Geophysical Prospecting, vol. 60, no. 3, pp. 444-465, 2012.

[19] L. A. Romero, D. C. Ghiglia, C. C. Ober, and S. A. Morton, "Phase encoding of shot records in prestack migration," Geophysics, vol. 65 , no. 2 , pp. $426-436,2000$.

[20] G. T. Schuster, X. Wang, Y. Huang, W. Dai, and C. Boonyasiriwat, "Theory of multisource crosstalk reduction by phase-encoded statics," Geophysical Journal International, vol. 184, no. 3, pp. 1289-1303, 2011.

[21] P. Temme, "A comparison of common-midpoint, single-shot, and planewave depth migration," Geophysics, vol. 49, no. 11, pp. 1896-1907, 1984.

[22] H. J. Tieman, "Improving plane-wave decomposition and migration," Geophysics, vol. 62, no. 1, pp. 195-205, 1997.

[23] X. Jia, B. Chen, and Q. Li, "A bipolar-bisection piecewise encoding scheme for multi-source reverse time migration," Communications in Computational Physics, vol. 28, no. 2, pp. 723-742, 2020.

[24] X. Jia, W. Chen, and B. Chen, "An efficient super-virtual shot encoding scheme for multisource reverse time migration," Geophysics, vol. 85, 2020.

[25] J. Wu and M. Bai, "Incoherent dictionary learning for reducing crosstalk noise in least-squares reverse time migration," Computers \& Geosciences, vol. 114, pp. 11-21, 2018.

[26] S.-J. Lee, T. Chen, L. Yu, and C.-H. Lai, "Image classification based on the boost convolutional neural network," IEEE Access, vol. 6, pp. 12755$12768,2018$.

[27] F. Milletari, N. Navab, and S.-A. Ahmadi, "V-net: Fully convolutional neural networks for volumetric medical image segmentation," in 2016 fourth international conference on $3 D$ vision (3DV), pp. 565-571, IEEE, 2016.

[28] S. Ren, K. He, R. Girshick, X. Zhang, and J. Sun, "Object detection networks on convolutional feature maps," IEEE transactions on pattern analysis and machine intelligence, vol. 39, no. 7, pp. 1476-1481, 2016.

[29] K. Zhang, W. Zuo, and L. Zhang, "Ffdnet: Toward a fast and flexible solution for cnn-based image denoising," IEEE Transactions on Image Processing, vol. 27, no. 9, pp. 4608-4622, 2018.

[30] D. Liu, W. Wang, X. Wang, C. Wang, J. Pei, and W. Chen, "Poststack seismic data denoising based on 3-d convolutional neural network," IEEE Transactions on Geoscience and Remote Sensing, vol. 58, no. 3, pp. 1598-1629, 2019.

[31] H. Wang, Y. Li, and X. Dong, "Generative adversarial network for desert seismic data denoising," IEEE Transactions on Geoscience and Remote Sensing, vol. 59, no. 8, pp. 7062-7075, 2020.

[32] X. Wang and J. Ma, "Adaptive dictionary learning for blind seismic data denoising," IEEE Geoscience and Remote Sensing Letters, vol. 17, no. 7, pp. 1273-1277, 2019.

[33] W. Li and J. Wang, "Residual learning of cycle-gan for seismic data denoising," IEEE Access, vol. 9, pp. 11585-11597, 2021.

[34] C. Guo, T. Zhu, Y. Gao, S. Wu, and J. Sun, "Aenet: Automatic picking of p-wave first arrivals using deep learning," IEEE Transactions on Geoscience and Remote Sensing, vol. 59, no. 6, pp. 5293-5303, 2020.

[35] Z. E. Ross, M.-A. Meier, and E. Hauksson, "P wave arrival picking and first-motion polarity determination with deep learning," Journal of Geophysical Research: Solid Earth, vol. 123, no. 6, pp. 5120-5129, 2018.

[36] S. Han, Y. Liu, Y. Li, and Y. Luo, "First arrival traveltime picking through 3-d u-net," IEEE Geoscience and Remote Sensing Letters, 2021.
[37] W. Zhang and J. Gao, "High-resolution velocity model building based on common-source migration images and convolutional neural networks," IEEE Geoscience and Remote Sensing Letters, 2021.

[38] F. Yang and J. Ma, "Deep-learning inversion: A next-generation seismic velocity model building method," Geophysics, vol. 84, no. 4, pp. R583R599, 2019.

[39] M. Araya-Polo, J. Jennings, A. Adler, and T. Dahlke, "Deep-learning tomography," The Leading Edge, vol. 37, no. 1, pp. 58-66, 2018.

[40] B. Wu, D. Meng, L. Wang, N. Liu, and Y. Wang, "Seismic impedance inversion using fully convolutional residual network and transfer learning," IEEE Geoscience and Remote Sensing Letters, vol. 17, no. 12, pp. 2140-2144, 2020.

[41] X. Wu, S. Yan, Z. Bi, S. Zhang, and H. Si, "Deep learning for multidimensional seismic impedance inversion," Geophysics, vol. 86, no. 5, pp. R735-R745, 2021.

[42] X. Wu, Y. Shi, S. Fomel, L. Liang, Q. Zhang, and A. Z. Yusifov, "Faultnet3d: Predicting fault probabilities, strikes, and dips with a single convolutional neural network," IEEE Transactions on Geoscience and Remote Sensing, vol. 57, no. 11, pp. 9138-9155, 2019.

[43] Z. Bi, X. Wu, Z. Geng, and H. Li, "Deep relative geologic time: a deep learning method for simultaneously interpreting 3-d seismic horizons and faults," Journal of Geophysical Research: Solid Earth, p. e2021JB021882.

[44] Y. Shi, X. Wu, and S. Fomel, "Saltseg: Automatic 3d salt segmentation using a deep convolutional neural network," Interpretation, vol. 7, no. 3 , pp. SE113-SE122, 2019.

[45] G. Matharu, W. Gao, R. Lin, Y. Guo, M. Park, and M. D. Sacchi, "Simultaneous source deblending using a deep residual network," in $S E G$ 2019 Workshop: Mathematical Geophysics: Traditional vs Learning, Beijing, China, 5-7 November 2019, pp. 13-16, Society of Exploration Geophysicists, 2020.

[46] S. Zu, J. Cao, S. Qu, and Y. Chen, "Iterative deblending for simultaneous source data using the deep neural network," Geophysics, vol. 85, no. 2, pp. V131-V141, 2020.

[47] A. Richardson and C. Feller, "Seismic data denoising and deblending using deep learning," arXiv preprint arXiv:1907.01497, 2019.

[48] O. Ronneberger, P. Fischer, and T. Brox, "U-net: Convolutional networks for biomedical image segmentation," in International Conference on Medical image computing and computer-assisted intervention, pp. 234 241, Springer, 2015.

[49] Z. Geng, X. Wu, Y. Shi, and S. Fomel, "Deep learning for relative geologic time and seismic horizons," Geophysics, vol. 85, no. 4, pp. WA87WA100, 2020.

[50] Z. Geng, Z. Zhao, Y. Shi, X. Wu, S. Fomel, and M. Sen, "Deep learning for velocity model building with common-image gather volumes," Geophysical Journal International, vol. 228, no. 2, pp. 1054-1070, 2021.

[51] K. He, X. Zhang, S. Ren, and J. Sun, "Deep residual learning for image recognition," in Proceedings of the IEEE conference on computer vision and pattern recognition, pp. 770-778, 2016.

[52] I. Laina, C. Rupprecht, V. Belagiannis, F. Tombari, and N. Navab, "Deeper depth prediction with fully convolutional residual networks," in 2016 Fourth international conference on $3 D$ vision (3DV), pp. 239248, IEEE, 2016.

[53] X. Wu, Z. Geng, Y. Shi, N. Pham, S. Fomel, and G. Caumon, "Building realistic structure models to train convolutional neural networks for seismic structural interpretation," Geophysics, vol. 85, no. 4, pp. WA27WA39, 2020.

[54] G. S. Martin, R. Wiley, and K. J. Marfurt, "Marmousi2: An elastic upgrade for marmousi," The leading edge, vol. 25, no. 2, pp. 156-166, 2006.

[55] M. D. Bloice, P. M. Roth, and A. Holzinger, "Biomedical image augmentation using augmentor," Bioinformatics, vol. 35, no. 21, pp. 4522-4524, 2019.

[56] P. Bojanowski, A. Joulin, D. Lopez-Paz, and A. Szlam, "Optimizing the latent space of generative networks," arXiv preprint arXiv:1707.05776, 2017.

[57] A. Paszke, S. Gross, F. Massa, A. Lerer, J. Bradbury, G. Chanan, T. Killeen, Z. Lin, N. Gimelshein, L. Antiga, et al., "Pytorch: An imperative style, high-performance deep learning library," Advances in neural information processing systems, vol. 32, pp. 8026-8037, 2019.

[58] D. P. Kingma and J. Ba, "Adam: A method for stochastic optimization," arXiv preprint arXiv:1412.6980, 2014. 